\title{
Densité osseuse des crêtes prémaxillaires reconstituées par apposition d'os cortical membraneux et intérêt de la chirurgie implantaire guidée
}

\author{
K. Bayet (Paris), B. Philippe (Paris)
}

Introduction : La procédure chirurgicale implantaire en présence d'os de densité hétérogène présente de réelles difficultés techniques en raison de la déviation des instruments de forage, de taraudage et d'insertion depuis l'os de densité élevée vers l'os de densité faible. L'objectif de cette communication consiste à valider la pertinence de la chirurgie guidée en présence de crêtes prémaxillaires reconstituées par apposition d'os autogène cortical membraneux en raison de l'importante différence de densité constatée entre le site receveur et le greffon.

Matériel et méthodes : Une étude rétrospective observationnelle est réalisée sur 20 scanners de cas cliniques ayant bénéficié d'appositions vestibulaires membraneuses (10 appositions d'os pariétal et 10 appositions d'os mandibulaire postérieur). L'ensemble des scanners ont été réalisés entre le 5eme et le 7 eme mois post-opératoire (coupes axiales de 0,625 mm espacées de 0,5 mm. Scanner Low Dose GE Optima). Toutes les analyses de densité ont été réalisées à l'aide du logiciel SIMPLANT PRO 17 (Dentsplysirona).

Résultat : La densité osseuse moyenne observée au sein des greffes pariétales est de 1798,20 UH. Celle observée au sein des greffes mandibulaire postérieure est de 1956,80 UH. L'os spongieux du site receveur maxillaire présente quant à lui une densité moyenne de 560 UH. Cette étude densitométrique confirme au prémaxillaire la différence de densité significative entre l'os greffé par apposition vestibulaire et l'os résiduel de la crête atrophiée (3.3 pour l'os pariétal et 3.4 pour l'os mandibulaire postérieur).

Discussion : Ces résultats confirment l'importante différence des densités au sein des crêtes fines reconstruites à l'aide d'os cortical membraneux. Compte-tenu des phénomènes provoqués de déviation des instruments de forage, de taraudage et d'insertion, la mise en place des implants doit être réalisée en chirurgie guidée afin d'assurer le contrôle exact de leur positionnement et de leur axe d'insertion. Bayet.kinz@gmail.com 